\title{
Effects of processing history on the evolution of surface damage layer and dislocation substructure in large grain niobium cavities
}

\author{
D. Kang and T. R. Bieler \\ Michigan State University, East Lansing, Michigan 48824, USA \\ C. Compton \\ Facility for Rare Isotope Beams, East Lansing, Michigan 48824, USA
}

(Received 27 August 2014; revised manuscript received 9 November 2015; published 16 December 2015)

\begin{abstract}
Large grain niobium $(\mathrm{Nb})$ is being investigated for fabricating superconducting radiofrequency cavities as an alternative to the traditional approach using fine grain polycrystalline $\mathrm{Nb}$ sheets. Past studies have identified a surface damage layer on fine grain cavities due to deep drawing and demonstrated the necessity for chemical etching on the surface. However, the origin of and depth of the damage layer are not well understood, and similar exploration on large grain cavities is lacking. In this work, electron backscatter diffraction (EBSD) was used to examine the cross sections at the equator and iris of a half cell deep drawn from a large grain $\mathrm{Nb}$ ingot slice. The results indicate that the damage (identified by a high density of geometrically necessary dislocations) depends on crystal orientations, is different at the equator and iris, and is present through the full thickness of a half cell in some places. After electron backscatter diffraction, the specimens were heat treated at $800^{\circ} \mathrm{C}$ or $1000^{\circ} \mathrm{C}$ for two hours, and the same areas were reexamined. A more dramatic decrease in dislocation content was observed at the iris than the equator, where some regions exhibited no change. The specimens were then etched and examined again, to determine if the subsurface region behaved differently than the surface. Little change in the dislocation substructure was observed, suggesting that the large grain microstructure is retained with a normal furnace anneal.
\end{abstract}

DOI: 10.1103/PhysRevSTAB.18.123501

PACS numbers: 61.05.J-, 61.72.Ff, 81.40.Gh, 81.65.Ps

\section{INTRODUCTION}

High purity niobium $(\mathrm{Nb})$ has been used to fabricate superconducting radiofrequency (SRF) cavities for particle accelerators over the past couple decades. SRF cavities can be formed by deep drawing slices directly cut from $\mathrm{Nb}$ ingots with large grains, as an alternative to the wellestablished technique using rolled fine grain polycrystalline $\mathrm{Nb}$ sheets. Thus far similar accelerating gradients have been achieved from both approaches [1-3]. Large grain ingot slices deform less uniformly than fine grain sheets, leading to problems such as thickness variations and ridges at grain boundaries in deep drawing, which may affect cavity performance. There are ongoing interests in the SRF community in relating the unique forming and metallurgical properties of large grain $\mathrm{Nb}$ to cavity performance $[4,5]$.

The deformation behavior of large grain $\mathrm{Nb}$ in deep drawing is complicated and current understanding is incomplete. Baars et al. assessed the effect of crystal orientation on deformation uniformity by studying uniaxial tensile

\footnotetext{
*Corresponding author. bieler@egr.msu.edu

Published by the American Physical Society under the terms of the Creative Commons Attribution 3.0 License. Further distribution of this work must maintain attribution to the author $(s)$ and the published article's title, journal citation, and DOI.
}

deformation of $\mathrm{Nb}$ single crystals directly extracted from an ingot [6]. Another relevant issue is the surface damage due to the friction effects from the die in deep drawing. Kneisel et al. identified the dependence of achievable accelerating gradients on the amount of material removed from a cavity surface [5,7] (Fig. 1). The thickness of the surface damage layer was estimated to be 100-200 $\mu \mathrm{m}$. Therefore, removing surface material from deep drawn cavities has become normative for the SRF community. However, the underlying mechanisms for surface damage have not been clearly identified. Besides, the prior study was done on fine grain cavities, and large grain cavities provide a method to assess surface damage, since there are few boundaries that obscure the surface damage effects.

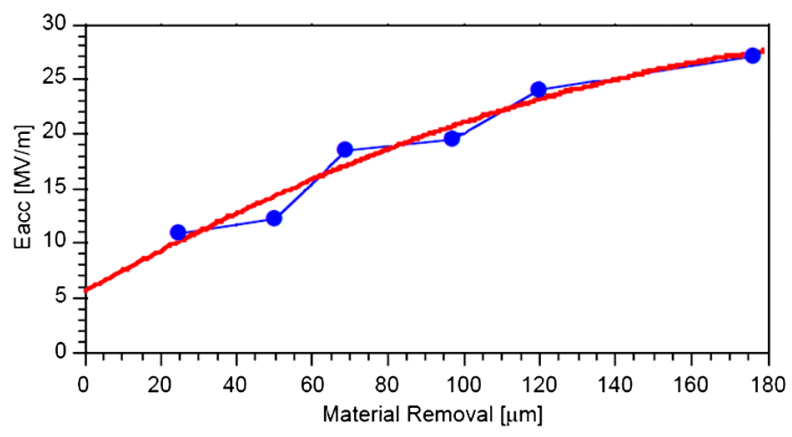

FIG. 1. Dependence of achievable gradients on the amount of material removed from the surface of a fine grain cavity [7]. 
Dislocations in a crystalline material exist in two forms $[8,9]$. Geometrically necessary dislocations (GND) account for the lattice curvature arising from an unbalanced population of dislocations of one sign within a grain. Such orientation gradients can be revealed by plotting a local average misorientation (LAM) map, in which larger LAM values correspond to higher GND content. Statistically stored dislocations (SSD) are slightly displaced dislocation pairs of opposite signs that do not contribute to the net orientation gradient. When there are adequate driving forces such as an elevated temperature, neighboring SSDs with opposite signs can move towards each other and annihilate. SSDs are not revealed by LAM maps.

Romanenko et al. identified a correlation between large LAM values and the "hot" regions of cavities [10,11]. The temperature increase from local heating increases the fraction of normally conducting electrons, so any potential sources for the heating need to be minimized. A proposed explanation for the local heating is that dislocations vibrate with passing phonons and disperse them, thus slow down thermal conduction [4]. Dislocations can also affect cavity performance if they pin magnetic flux centers, resulting in irreversibility of magnetization curves [5]. These adverse effects of dislocations necessitate a heat treatment for recovery or recrystallization after deep drawing.

SRF cavities are typically given a heat treatment after they are welded together. Various schedules have been used in the SRF community [12,13], such as $600^{\circ} \mathrm{C}$ for 10 hours, $800^{\circ} \mathrm{C}$ for two hours, and $1000^{\circ} \mathrm{C}$ for two hours, and no consensus has yet been reached as to which one is the most beneficial for cavity performance. While it is commonly understood that heat treatments eliminate dislocations by mechanisms of recovery/recrystallization, it is desirable to obtain a more quantitative understanding about dislocation removal. Also, Chandrasekaran et al. found that a heat treatment temperature above $800^{\circ} \mathrm{C}$ usually leads to significant recovery of the phonon peak on strained single crystal and bicrystal specimens, and that $1000{ }^{\circ} \mathrm{C}$ may be an optimal temperature for recovering the phonon peak $[14,15]$.

A thin and stable pentoxide layer tends to form on the surface of $\mathrm{Nb}$ upon air or water exposure. While the oxide itself does not greatly degrade the superconducting properties of $\mathrm{Nb}$, the interface between the oxide layer and $\mathrm{Nb}$ can absorb contaminants such as hydrogen [16-18]. It has been observed that a vacuum heat treatment at above $500{ }^{\circ} \mathrm{C}$ is effective in dissolving the surface oxide into the bulk [19].

In this paper (an extended version of [20] presented at SRF 2013, with updated results and a more detailed discussion), the development of dislocation substructure was examined using electron backscatter diffraction (EBSD) on specimens extracted from excess material removed from large grain cavity halves at the equator and iris. Following typical cavity fabrication processes, the deformed samples were heat treated at $800^{\circ} \mathrm{C}$ or $1000^{\circ} \mathrm{C}$ for two hours and characterized again after the heat treatment. Since cavities are commonly etched lightly after the heat treatment, the surface was similarly etched to assess whether removing a thin surface layer revealed any difference in the dislocation substructure.

\section{MATERIALS AND METHODS}

Samples were cut from two rings trimmed from the equator and iris using electrodischarge machining (EDM) of a large grain cavity half cell prepared at Thomas Jefferson National Accelerator Facility (Newport News, VA). Two specimens were extracted from the equator ring, and one from the iris ring, as shown in Fig. 2. All three specimens contained a grain boundary and geometrical earing (heterogeneous deformation) features associated with the grain boundary, where the resistance to thinning resulted in more of a drag force. The cross sections of the specimens represent surfaces that would eventually be welded to other half cells. These surfaces were hand ground using $\mathrm{SiC}$ abrasive paper to remove the EDM recast layer. The relatively smooth EDM finish enabled use of fine abrasive paper to minimize damage from grinding, starting with grit 1200 (particle size $\sim 15.3 \mu \mathrm{m}$ ) and ending with grit 4000 (particle size $\sim 2.5 \mu \mathrm{m}$ ) [21]. After grinding, the specimens were given a light chemical etch that removed another $10 \mu \mathrm{m}$ of material to provide a surface suitable for EBSD analysis using a scanning electron microscope. The buffered chemical polishing (BCP) solution was a mixture of phosphoric acid, hydrofluoric acid and nitric acid in the ratio of $1: 1: 2$, which is commonly used for SRF cavities [22].

EBSD is a well-established technique for acquiring crystal orientations, in which electron beams are focused onto a flat crystalline sample whose surface normal is tilted

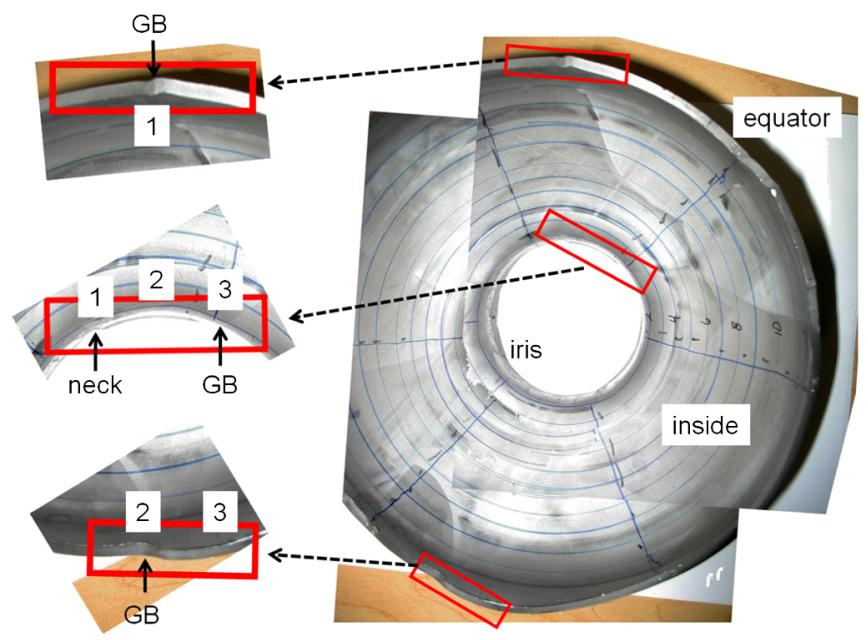

FIG. 2. Locations where the three specimens were extracted from a half cell provided by Jefferson Lab. Grain boundaries (GB) and a neck are indicated in the blown-up images on the left. Numbers mark roughly where each EBSD scan was done on the equator/iris (in cross reference with Fig. 4). The concave side is the inside of a cavity, and the opposite is the outside. 
$70^{\circ}$ from the beam axis. Electrons scattered by the sample in the backward directions form intersecting bands (Kikuchi patterns) on a fluorescent screen (the EBSD detector). Through a Hough transform, these patterns can be converted into crystal orientations [23,24]. In this paper, a CamScan 44 FE scanning electron microscope operated at $20 \mathrm{kV}$ was used for acquiring EBSD data, with a working distance of $33 \mathrm{~mm}$ and an exposure time of $0.04 \mathrm{~s}$ for each pattern. The step size used for all scans was $20 \mu \mathrm{m}$. The data were cleaned up using a single iteration of grain dilation and grain confidence index (CI) standardization with a minimum grain size of 3 , and pixels with $\mathrm{CI}>0.1$ were used to draw the orientation maps.

After EBSD measurements were made, the iris specimen, and one of the two equator specimens were given an $800^{\circ} \mathrm{C} / 2 \mathrm{~h}$ heat treatment in a dedicated vacuum furnace for $\mathrm{Nb}$, while the other equator specimen was heat treated at $1000^{\circ} \mathrm{C} / 2 \mathrm{~h}$. For the $1000^{\circ} \mathrm{C}$ heat treatment, titanium was used as a getter to minimize uptake of hydrogen and oxygen into $\mathrm{Nb}$. The same areas were scanned again to identify the effects of heat treatment on the surface damage layer. After that, the specimens were etched using BCP to remove another $10 \mu \mathrm{m}$ material from the surface, and the same areas were examined again to identify changes that occurred in the subsurface region.

Various analyses can be performed with an EBSD data set, one of which is based on the LAM method. For a given point, LAM reports the average misorientations between all of its neighboring points surrounding a specified kernel, as illustrated in Fig. 3 for the second nearest neighbor sampling area [25].

LAM results can be presented in the form of a gray scale map or a histogram. In an LAM map, each EBSD data point is assigned a certain shade of gray between white and black, corresponding to misorientations from $0^{\circ}$ to a user defined maximum value. In an LAM histogram, the angular range of misorientations is divided into small user defined bins, and the number of misorientations in each bin is counted, which then determines the number fraction for each bin.

The image quality (IQ) of EBSD patterns is another indicator of defect content [25]. The IQ parameter is the sum of detected peaks in the Hough transform of a pattern,

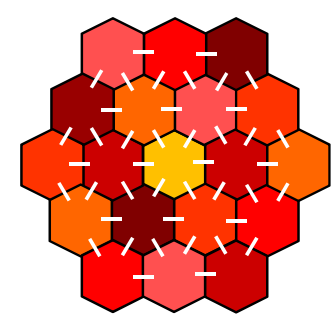

FIG. 3. LAM calculations using the second nearest neighbor sampling area-for the point at the center, average the misorientations between all of its neighboring points connected by the short white lines surrounding the kernel [25]. which is a component of indexing EBSD patterns. Any distortions to the lattice within the diffracting volume will produce diffraction patterns with lower IQ. As the most dominant type of defects in a crystal, dislocations can be qualitatively assessed by an IQ map, where darker areas indicate a higher dislocation density. However, IQ is affected by both GNDs and SSDs while LAM only reflects GNDs. Other factors such as grain boundaries and surface contamination will also reduce IQ. Furthermore, IQ can be affected by EBSD imaging parameters that are often different for each scan, may vary smoothly over the area of the scan, and will differ from grain to grain (due to different amounts of backscatter electrons). Thus, the IQ map is informative when interpreted as a relative quantity within a given scan, but less so when comparing between scans.

Similar to LAM maps, IQ maps are presented in a gray scale that varies linearly from black, the minimum IQ on a map, to white, the maximum IQ on a map-which differs for each scan. In the following section, both LAM and IQ maps will be used to assess total defect content.

\section{RESULTS AND DISCUSSION}

Figure 4 shows the secondary electron images for the six cross-sectional areas where the EBSD scans were performed. The specimens were mounted such that the outside of the cavity always faced upwards. Normal direction orientation maps, LAM maps and IQ maps are shown in Figs. 5 and 6 for the equator and iris, for the locations identified in Fig. 4, with the as-deformed maps above, annealed in the middle, and etched on the bottom for each region. On the orientation maps, low and high angle grain boundaries are marked by white $\left(3^{\circ}-15^{\circ}\right)$ and black $\left(>15^{\circ}\right)$ lines. The LAM maps are shown using the second nearest neighbor sampling area (as in Fig. 3) and a gray scale from white $\left(0^{\circ}\right)$ to black $\left(2^{\circ}\right)$. For easy comparison, orientation maps, LAM maps and IQ maps from matching areas are shown adjacent to each other.

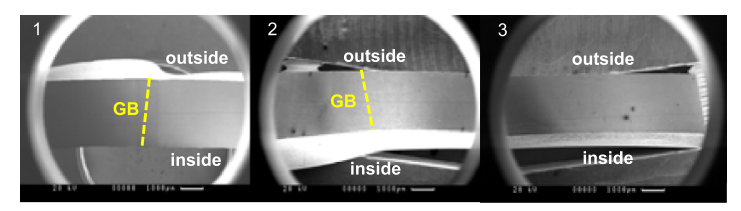

Areas (cross sections) scanned on the equator specimens

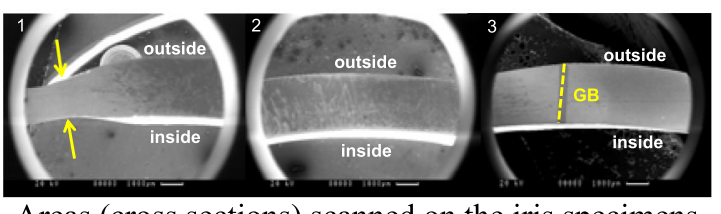

Areas (cross sections) scanned on the iris specimens

FIG. 4. Secondary electron images for the six cross-sectional areas examined by EBSD. Grain boundaries and the neck are marked in accordance with Fig. 2. The numbering is also the same as Fig. 2. 
Significant orientation gradients are evident by the large changes in color over distances of 100-200 $\mu \mathrm{m}$ in the orientation maps. The dark regions on the LAM maps near the surface indicates larger LAM values than the light regions in the bulk, which is evidence for surface damage. The orientation maps with grain boundaries show that the magnitude of damage depends on grain orientations. For example, Equator 2 in Fig. 5 shows that LAM values on the outside surface are greater in the right grain than in the left; the difference is even larger after heat treatment. The heat treatment reduced the magnitude of orientation gradients inside the material, as the color became lighter, which represents a more perfect crystal. However, it hardly changed the orientation gradients near the surface. From the IQ maps, surface contaminations, grain boundaries, and artifacts such as residual deformation from scratches are readily visible.

From the shape change in the iris and equator, the equivalent strains are different. The iris experienced hoop tension and bending that reached an equivalent strain of about $40 \%$. Based on continuum FEM simulations of the forming process for cavities with similar geometry [26], the equator experienced a more complex history involving hoop compression and bending to an equivalent strain of about $20 \%$. The difference between the equator and iris LAM maps in Figs. 5 and 6 is consistent with the larger strain in the iris; prior to heat treatment, the equator scans have large areas that are light gray, while the iris scans are dominated by darker shades of gray. However, the larger dislocation density in the iris provided a greater driving force for recovery, which can account for the more dramatic color change for the iris specimen after the heat treatment.

No evidence for recrystallization, i.e., emergence of new orientations, was observed after the heat treatment. This suggests that conventional furnace heating facilitates recovery and prevents recrystallization in deformed large grains. In contrast, previous studies on electron beam welding of deformed single crystals [4,27-29] showed that recrystallization occurred in the heat affected zone adjacent to the weld, leading to a different grain size on either side of the weld. The rapid heating rate apparently reached the recrystallization temperature before too much recovery took place, so that the stored dislocation defect energy was available for nucleation and growth of recrystallized grains.

Furthermore, high LAM values are located near the surface after heat treatment in all areas except on the outside of the iris to the left of the grain boundary in Iris 3, where the left grain was strained (thinned) more than the right grain. In the regions that did not initially have high surface LAM values, they remained unchanged after annealing (with the notable exception of Equator 2 in the left grain, where removal of $10 \mu \mathrm{m}$ revealed much higher LAM values).

The heat treatment also led to removal of defects on the initially polished surface in some cases, notably the orientation gradients that were the remnants of a scratch from left to right in Equator 2, which disappeared after the heat treatment. However, what appeared to be similar scratch features in Equator 3 just above the center did not completely disappear with heat treatment, and the same feature with a wider breadth of misorientation was present after removing $10 \mu \mathrm{m}$, which implies that this feature is a long range defect structure that formed during deformation and was stable through the heat treatment.

The grain boundary in Iris 1 in Fig. 6 apparently developed during deformation. This boundary has the highest misorientation at the inner surface, and disappears with distance toward the outer radius. Since the boundary has large LAM values as indicated by the dark color, it appears to be a result of polygonization [8], which lead to a low-energy configuration of dislocations where dislocations line up with each other with a regular spacing in a direction inclined to the slip plane. This implies that a larger bending strain occurred during deep drawing at this position to cause an unbalanced population of dislocations of one sign to form the low angle boundary.

The formation of the low angle boundary at this particular position of the iris may be a consequence of the changing driving force for dislocation activities with respect to the crystal orientation, as the hoop stress is in a different direction at every position in the original large grain. It is also possible that a preexisting low angle boundary became a trap for dislocation accumulation that led to the formation of a sharper boundary. Evidence for this possibility is visible in the Iris 2 maps, where a very low angle, nearly vertical boundary to the left of center is present. This boundary is evident as a subtle color change in the EBSD map in the same place in all three locations, but it is not evident in the as-deformed LAM map due to high LAM values that obscure the low angle boundary.

A close inspection of the orientation maps indicates that there is sometimes a slight migration of grain boundaries at each stage. For example, the jog in the boundary in Equator 1 in Fig. 5 is at a different location in the as-deformed and heat treated maps, and is not present in the etched map. This suggests that the driving force (primarily plastic strain) for recovery was not homogeneous, such that the defect removal occurred at different speeds in various parts of the material.

After BCP, the LAM values became higher in several cases, an example of which is shown in the upper third of the left grain in Equator 2 (Fig. 5), where many low angle boundaries are evident, as indicated by both the white low angle boundary lines in the EBSD map and the extensive black local maxima in the LAM map. Figure 7 provides a more quantitative visualization of this effect, in which the misorientation profiles for three corresponding lines in the same places for each of the three maps in Fig. 5 are plotted. In the after-BCP map, the profile for the left grain exhibits a large oscillation of about $2^{\circ}-3^{\circ}$ as the line crosses the area with lots of low angle grain boundaries. One possible explanation is that the recovery was less effective in the 

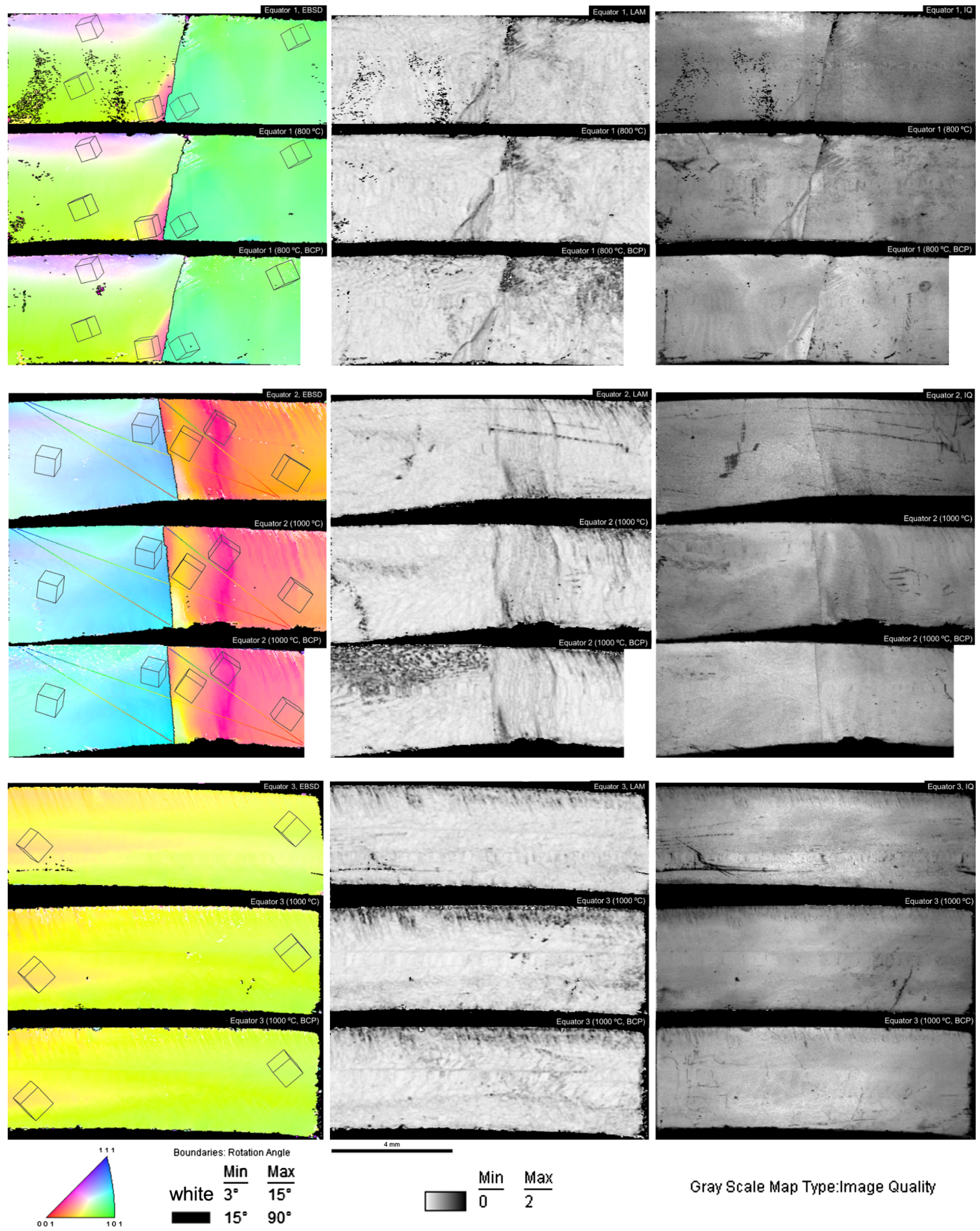

Gray Scale Map Type:Image Quality

FIG. 5. Normal direction orientation maps (left), LAM maps (middle), and IQ maps (right) for the three equator scans. For each area, results for as-deep drawn, after heat treatment, and after BCP are shown from top to bottom. Scale and legends are common. 

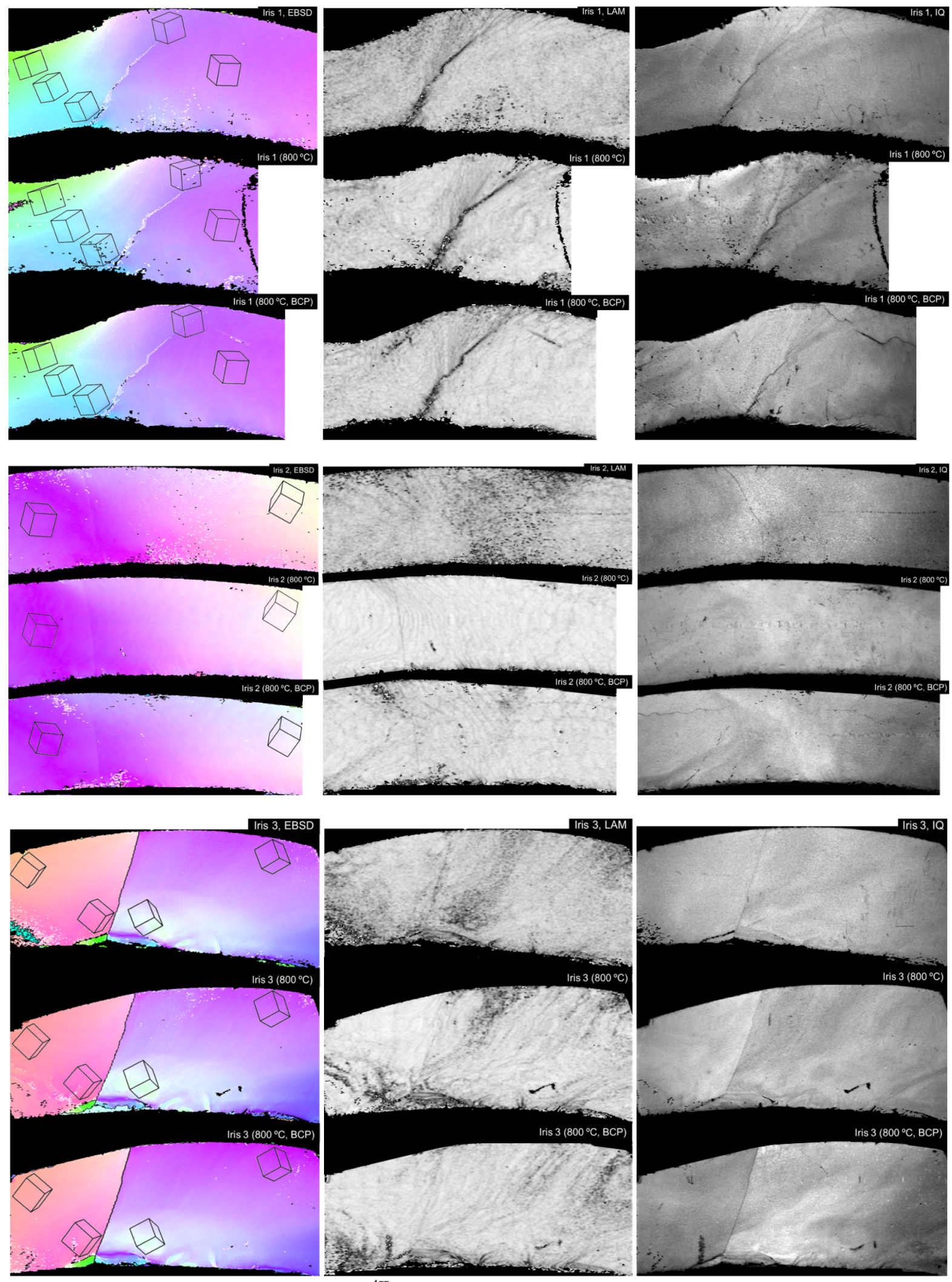

FIG. 6. Normal direction orientation maps (left), LAM maps (middle), and IQ maps (right) for the three iris scans. For each area, results for as-deep drawn, after heat treatment, and after BCP are shown from top to bottom. Scale is common and legends are the same as those in Fig. 5. 


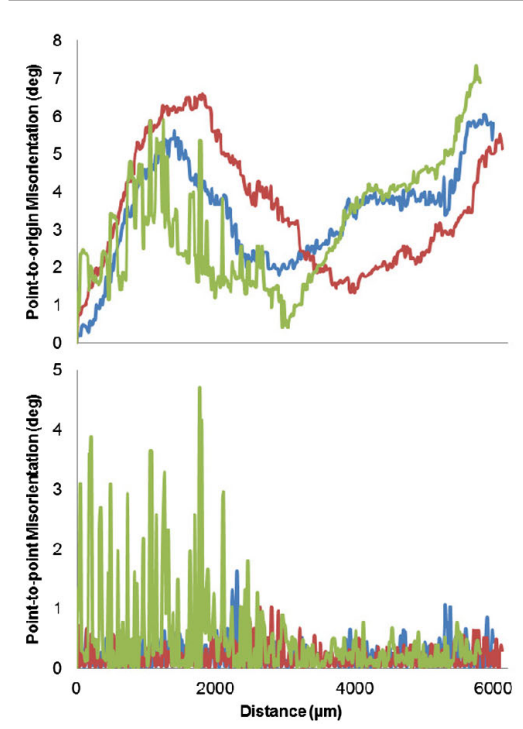

Left grain

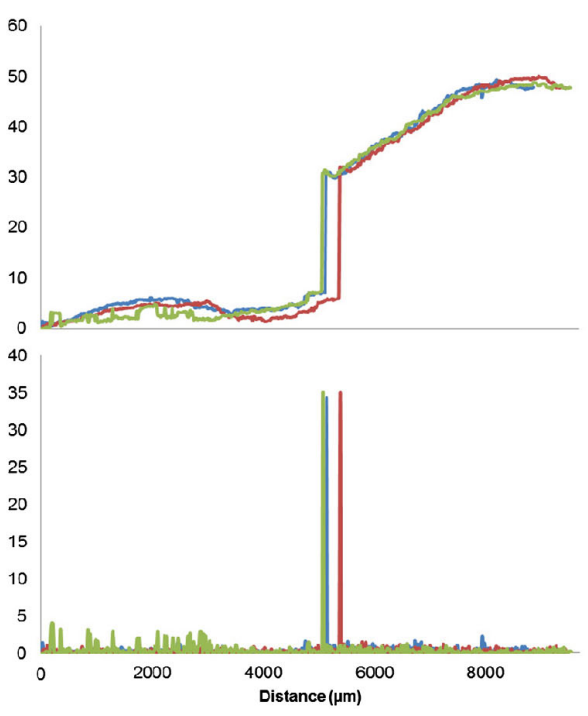

Across grain boundary

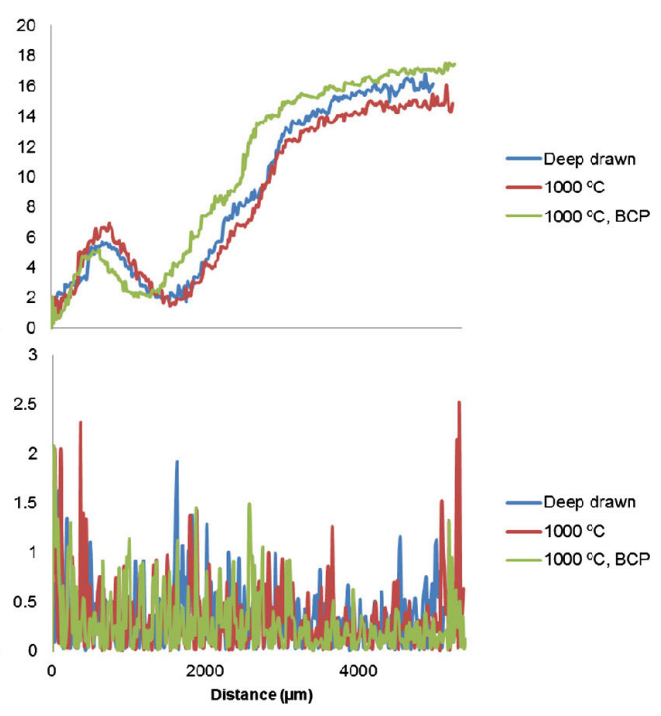

Right grain

FIG. 7. Point-to-origin (upper 3) and point-to-point (lower 3) misorientation profiles for the three colored lines drawn on Equator 2 in Fig. 5. The blue ends of the colored lines in Fig. 5 are at the origin.

subsurface region than on the surface, as the surface could serve as a sink for dislocations to exit. This effect is also evident in the after BCP LAM map of Iris 2 in Fig. 6.

Figure 8 shows the LAM histograms for the six scans after deep drawing, after heat treatment and after BCP. Before heat treatment, all iris scans (dashed lines) had larger LAM values than the equator scans (solid lines), which is consistent with the larger strain and the greater amount of dark colors in the as-deep drawn LAM maps. Heat treatment resulted in more dramatic changes in the iris than the equator, as all the LAM peaks for the iris scans moved to the left by about $0.25^{\circ}$. In contrast, the peak positions for the equator scans moved in both directions, suggesting that the recovery processes were highly variable. For example, after the $1000^{\circ} \mathrm{C} / 2 \mathrm{~h}$ heat treatment, the peak for Equator 3 moved to the right, indicating a greater amount of GND content. This may explain why the equator is more susceptible to forming hot spots in operating cavities [10], as there are apparently regions where the local misorientation actually gets higher after a heat treatment. This is also consistent with observations in [15], where a larger strain is usually necessary for a more complete recovery of phonon peaks after heat treatment. After BCP, the LAM values showed no consistent relationship with the as-heat treated values, as a different volume of material was sampled. These histograms are more similar to each other, which may reflect being distant from a free surface that provides more degrees of freedom for changes to occur.

The IQ maps provide a contrast to LAM maps, as they show the sum of all defect content, including impurities, vacancies, GNDs and SSDs. In the LAM maps, there is clear evidence for cell boundaries, where there are distinct networks within which there are few GNDs. The IQ maps generally show lower IQ (darker regions) where there is higher GND content, but the cell structures are not evident. This suggests that the SSDs and other defects overwhelm the GND contribution. Annealing affects the IQ maps in a way that parallels the GND content, but there are also

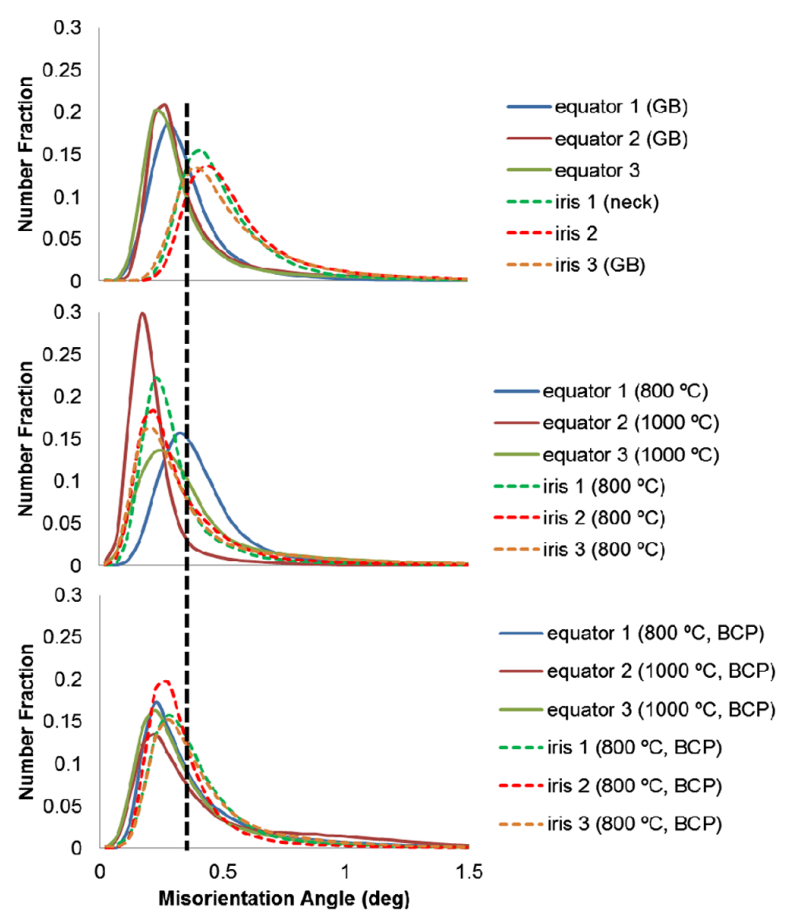

FIG. 8. LAM histograms of the six EBSD scans for as-deep drawn (top), after annealing (middle), and after BCP (bottom). Horizontal scale is common. 

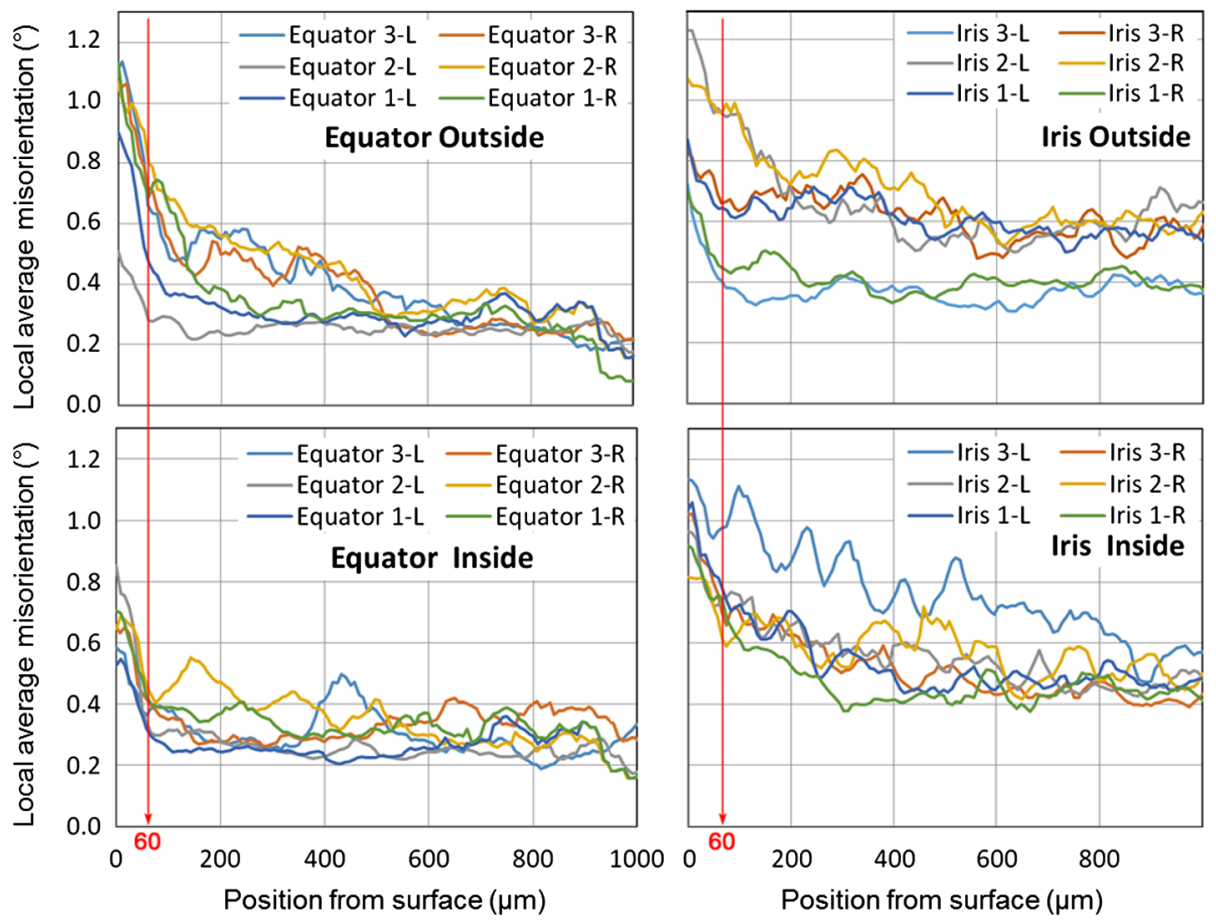

FIG. 9. Local average misorientation (LAM) profiles in the six grains obtained from averaged traces on the inside and outside of the equator and the iris.

regions of high IQ that correspond with high LAM values in most maps.

The depth of surface damage can be estimated from the LAM maps, as LAM values correspond with GND content (lattice curvature). To evaluate this, multiple traces from the surface inward in the six grains were extracted and averaged. The LAM profiles for the six areas in the equator (left and right on each scan) and six areas in the iris are plotted with depth from the surface in Fig. 9. All six grains responded differently to forming as evident by variations in the plot. LAM values for the iris are in general higher than the equator, consistent with prior observations. At the equator, LAM values come to a low and constant value at a smaller depth than the iris, again indicating that the defect content is greater at the iris. The inside and outside behaved differently as well-the equator inside and iris outside reach a constant LAM value at $\sim 60 \mu \mathrm{m}$, while the LAM values for the equator outside and iris inside have a more gradual drop from the surface.

Significant defect content is present at depths up to $\sim 200 \mu \mathrm{m}$ at several locations. This may explain why a $\sim 70 \mu \mathrm{m} \mathrm{BCP}$ on the subsequently fabricated cavity did not initially yield good performance, but it improved significantly after a second $\sim 170 \mu \mathrm{m}$ surface removal by centrifugal barrel polishing, BCP, and electropolishing. ${ }^{1}$ This suggests that these high LAM values near the surface can be understood as a metric for damage. Comparing with fine

\footnotetext{
${ }^{1}$ Further details of cavity performance tests performed by
} Ciovati will be presented in a future paper. grain cavity results in Fig. 1, it appears that because cavity performance was still improving with $180 \mu \mathrm{m}$ of surface removal, that damage from forming occurs similarly in fine grain cavities, though it was estimated using a different approach [7]. A parallel study on a fine grain cavity would be needed for a direct comparison.

A limitation of this study is that EBSD only provides information about the surface, as the penetration depth for the electrons is less than $1 \mu \mathrm{m}$. Therefore, it remains an open question as to whether the effect of heat treatment on the original surfaces examined is a good representation of the bulk, as the surface removal demonstrates. In fact, the discrepancy in LAM values before and after BCP does suggest different recovery behavior between the surface and the bulk. In large grain material, it may be easier for dislocations near the surface to annihilate or exit during recovery. While the surface condition is important for superconductivity, thermal conductivity (a bulk effect) is also crucial, so it is equally important to know whether dislocations in the bulk have been effectively removed. A 3D x-ray study would help address this concern, and such data have been acquired and will be the subject of a future paper, for more insight into the microstructural evolution during the heat treatment.

Romanenko et al. reported that a low temperature bake at around $120^{\circ} \mathrm{C}$ caused a reduction in LAM values in both small and large grain cavities [11], which is somewhat striking, as it is generally believed that dislocations are hard to move much below the recovery temperature. In this paper, care was taken to observe exactly the same areas 
before and after the heat treatment and surface removal, showing that GNDs in large grain material are stable and difficult to remove even after the higher temperature anneal. In contrast, the data in [11] suggests that more of a change in LAM took place in large grain than small grain material, although there was no indication that the exact same regions were measured before and after the bake. In both studies, the trends show a shift of the LAM peaks to the left after heat treatment, but the variability may arise from the heterogeneity in dislocation substructures, so larger statistical sampling may be needed before a quantitative interpretation of the magnitude of the shift can be developed. Clearly, there are regions with very stable and entangled dislocation microstructures that are resistant to the recovery-based removal, and the heterogeneity of these regions may account for the occasional etch pits observed on large grain cavities that are sometimes associated with rf losses [30,31].

\section{CONCLUSIONS}

EBSD analyses on the iris and equator specimens indicate that the surface damage from deep drawing depends on crystal orientations, is different at the equator and iris, and is severe even in the bulk at the iris. The iris and equator respond differently to heat treatment due to their different post-deformation defect states. While the $800^{\circ} \mathrm{C} / 2 \mathrm{~h}$ may be sufficient for recovery near the iris, a higher temperature or longer time may be necessary for the equator to achieve a similar degree of dislocation removal. These heat treatments do not effectively remove networks of GNDs. The depth of surface damage may be similar for large grain and fine grain cavities, although further study is needed to verify this.

\section{ACKNOWLEDGMENTS}

The authors would like to thank G. Ciovati and G. Myneni from Jefferson Lab for supplying the samples used in this work. This work was supported by the U.S. Department of Energy, Office of High Energy Physics, through Grant No. DE-SC0009962.

[1] P. Kneisel, G. R. Myneni, G. Ciovati, J. Sekutowicz, and T. Carneiro, Development of large grain/single crystal niobium cavity technology at Jefferson Lab, AIP Conf. Proc. 927, 84 (2007).

[2] A. Ermakov, I. Jelezov, X. Singer, W. Singer, G. B. Viswanathan, V. Levit, H. L. Fraser, H. Wen, and M. Spiwek, Physical properties and structure of large grain/ single crystal niobium for superconducting rf cavities, J. Phys. Conf. Ser. 97, 012014 (2008).

[3] W. Singer et al., Development of large grain cavities, Phys. Rev. ST Accel. Beams 16, 012003 (2013).
[4] T. Bieler et al., Physical and mechanical metallurgy of high purity $\mathrm{Nb}$ for accelerator cavities, Phys. Rev. ST Accel. Beams 13, 031002 (2010).

[5] H. Padamsee, RF Superconductivity: Science, Technology, and Applications (WILEY-VCH Verlag GmbH \& Co. KGaA, Weinheim, 2009).

[6] D. Baars, Investigation of active slip systems in high purity single crystal niobium, Ph.D. dissertation, Michigan State University, 2013.

[7] P. Kneisel, Performance of $1300 \mathrm{MHz}$ KEK-type singlecell niobium cavities, Proceedings of the SRF97, RF superconductivity, Padua, 1997 (Joint Accelerator Conferences Website (JACoW), Padua, 1997), Vol. 3, pp. 463-471.

[8] D. Hull and D. J. Bacon, Introduction to Dislocations, 4th ed. (Butterworth-Heinemann, Oxford, 2001).

[9] L. Bortoloni and P. Cermelli, Statistically stored dislocations in rate-independent plasticity, Rend. Sem. Mat. 58, 25 (2000).

[10] A. Romanenko, Review of high field Q-slope, surface measurements, Proceedings of the SRF2007, Beijing, 2007 (Joint Accelerator Conferences Website (JACoW), Beijing, 2007), pp. 75-81.

[11] A. Romanenko and H. Padamsee, The role of near-surface dislocations in the high magnetic field performance of superconducting niobium cavities, Supercond. Sci. Technol. 23, 045008 (2010).

[12] G. Ciovati, P. Kneisel, and G. R. Myneni, America's Overview of Superconducting Science and Technology of Ingot Niobium,, AIP Conf. Proc. 1352, 25 (2011).

[13] P. Dhakal et al., Effect of high temperature heat treatments on the quality factor of a large-grain superconducting radio-frequency niobium cavity, Phys. Rev. ST Accel. Beams 16, 042001 (2013).

[14] S. Chandrasekaran, N. T. Wright, T. R. Bieler, and C. C. Compton, Effect of heat treatment temperature on thermal conductivity of large grain superconducting niobium, Proceedings of the SRF2011, Chicago, 2011 (Joint Accelerator Conferences Website (JACoW), Chicago, 2011), pp. 593-596.

[15] S. Chandrasekaran, T. R. Bieler, C. C. Compton, and N. T. Wright, Recovery of phonon peak in annealed niobium as a function of initial strain and hydrogen concentration, Proceedings of the Oral presentation at 7th SRF Materials Workshop, SRFMW2012, Newport News, (2012).

[16] C. Antoine, Materials and surface aspects in the development of SRF Niobium cavities, Report No. EuCARDBOO-2012-001.

[17] C. Antoine, A. Aspart, S. Regnault, and A. Chincarini, Surface studies: Method of analysis and results, Proceedings of the SRF2001, Tsukuba, 2001, (Joint Accelerator Conferences Website (JACoW), Tsukuba, 2001), pp. 272-278.

[18] R. Ricker and G. Myneni, Evaluation of the propensity of niobium to absorb hydrogen during fabrication of superconducting radio frequency cavities for particle accelerators, J. Res. Natl. Inst. Stand. Technol. 115, 353 (2010).

[19] R. Kirby et al., Residual oxygen on $\mathrm{Nb}$ heated to $500 \mathrm{C}$, Report No. SLAC-TN-05-049. 
[20] D. Kang, T. R. Bieler, and C. Compton, Surface damage and effects of heat treatment on large grain $\mathrm{Nb}$ cavities, Proceedings of the SRF2013, Paris, 2013 (Joint Accelerator Conferences Website (JACoW), Paris, 2013), pp. $455-460$.

[21] D. Zipperian, Silicon carbide abrasive grinding, Quality Matters Newsletter 1, 1 (2002).

[22] V. Palmieri, F. Stivanello, S. Y. Stark, C. Roncolato, and M. Valentino, Besides the standard niobium bath chemical polishing, Proceedings of the SRF2001, Tsukuba, 2011 (Joint Accelerator Conferences Website (JACoW), Tsukuba, 2001), pp. 408-412.

[23] O. Engler and V. Randle, Introduction to Texture Analysis: Macrotexture, Microtexture, and Orientation Mapping (CRC Press, Boca Raton, 2009), edition 2nd ed.

[24] K. Kunze, S. I. Wright, B. L. Adams, and D. J. Dingley, Advances in automatic EBSP single orientation measurements, Textures and microstructures 20, 41 (1993).

[25] EDAX-TSL Software V5.31 manual.
[26] M. Johnson, Facility for rare isotope beams (private communication).

[27] D. Baars, T. R. Bieler, A. Zamiri, F. Pourboghrat, and C. Compton, Crystal orientation effects during fabrication of single or multi-crystal Nb SRF cavities, Proceedings of the SRF2007, Beijing, 2007 (Joint Accelerator Conferences Website (JACoW), Beijing, 2007), pp. 111-115.

[28] D. Baars, H. Jiang, T. Bieler, C. Compton, P. Bauer, and T. Grimm, Crystal orientations near welds in high RRR niobium with very large grains, IEEE Trans. Appl. Supercond. 17, 1295 (2007).

[29] D. Baars, H. Jiang, T. R. Bieler, A. Zamiri, F. Pourboghrat, and C. Compton, Effects of elastic modulus on deformation and recrystallization of high purity $\mathrm{Nb}$, Ceramic Transactions 201, 391 (2008).

[30] X. Zhao, G. Ciovati, and T. R. Bieler, Characterization of etch pits found on a large-grain bulk niobium superconducting radio-frequency resonant cavity, Phys. Rev. ST Accel. Beams 13, 124701 (2010).

[31] G. Ciovati et al. (unpublished). 